\title{
Modélisation du trajet global de la flèche avec différents types d'équipements
}

\author{
- Rapport de recherche -
}

\author{
Daniel DINU • Nicolas HOUEL • Marc DELLENBACH
}

\begin{abstract}
Gill in archery directly depends on the interaction between the
subject, the bow and the arrow when shooting. Researches in biomechanics of archery have frequently described the different coordination and muscular activation during the shoot of the subject. Few of them describe the influence of the bow (mechanical characteristics, stabilizers, etc.) on the subject's skill and physical capacities. The aim of the study is to compare the influence of two bows on the subject's skill. An international level archer shot a sequence of twelve arrows (A1) X10, 380 (Easton) and a sequence of twelve arrows (A2) X10 Pro Tour, 380 (Easton). Each sequence of arrows were shot with two bows (B1: 27 inch Inno carbon bow handle associated with 70 inch and $38 \mathrm{lb}$ bow limb; B2 : 25 inch Inno bow carbon handle associated with 70 inch and $40 \mathrm{lb}$ bow limb). All archers' shots were performed on a $70 \mathrm{~m}$ distant target. For each arrow, heights at shot (H1) and on the target (H2) were measured. The velocity (km.h-1) of the arrow was recorded using Stalker ATS radar when it left the bow. The angle $(\alpha)$ between the arrow and the horizontal axis was estimated using the previous measurement and the Newton's law. Multi-varied Anova test was used to define the influence of bows and arrows on the shot parameter $(V)$ and archer's skill $(\alpha, H 2)$. Results show that the use of the B2A2 condition would be the less interesting for this archer's skill in order to perform the centre of the target. Indeed, H2 with $\mathrm{B} 2 \mathrm{~A} 2$ condition is usually $10 \mathrm{~cm}$ above the centre of the target. The use of the $\mathrm{B} 2 \mathrm{~A} 1$ condition could permit the archer to perform the centre of the target as accurately as the $\mathrm{B} 1$ conditions. When using $\mathrm{B} 2 \mathrm{~A} 1$ condition, a shot is realized with smaller velocity (219 \pm 0.3 versus $226 \pm 0 \mathrm{~km} . \mathrm{h}-1)$ and bigger angle $\alpha$ ( $5.45 \pm 0.3$ versus $5.16 \pm 0.03$ degree). For this archer, B2A1 condition could help him to reach the centre of the target with less energy to bend the bow and shoot with less velocity, and bigger range of angle when shooting (0.6 versus 0.08 degree).
\end{abstract}

Keywords: archery, skill, arrows, bow 


\section{Introduction}

L'élégance du geste du tir à l'arc a depuis longtemps exercé une grande fascination. De part sa complexité et son esthétisme, le tir à l'arc est devenue le sport par excellence dans l'Egypte antique. A cette époque les archers visaient notamment des lingots de cuivre.

Plus tard, le philosophe allemand Eugen Herrigel qui étudie le tir à l'arc japonais traditionnel, relève cette discipline au rang d'art (Rousseau \& Nicolier, 2005). En effet, sa démarche s'inscrivait dans une logique liée à la pratique d'un instrument de musique: le violon. Violon et tir à l'arc, le mariage peut sembler à priori surprenant...et pourtant Herrigel avait défini ce rapprochement entre les deux disciplines comme la capacité commune dans les deux cas, à imprimer sa volonté à un objet. D'après lui la pratique du tir à l'arc et les techniques qui s'y rattachent devaient nourrir la pratique de la musique.

Avec l'ère moderne, cette discipline, devenue olympique, a déjà été l'objet d'études émanant principalement du monde sportif. Ces analyses, s'appuyant souvent sur l'observation directe, décrivent les séquences gestuelles des archers et la manière dont les meilleurs compétiteurs les enchaînent (Avon-Coffrant, 1977; Frederic, 1979; Hunt et coll. 1987; Leroyer \& Le Gaverec, 1991; Nayrole \& Simon, 1990; Ferriou et coll. 1992).

Un nombre plus restreint d'études utilisant des techniques d'investigation plus précises ont permis d'aborder le geste du tir à l'arc d'un point de vue biomécanique. En effet, en 1998 Kooi et coll., appréhendent l'interaction entre l'arc et la flèche par la modélisation du mouvement de la flèche sur la poignée de l'arc. Ils concluent que la modélisation de la dynamique de la flèche avant le tir effectif peut contribuer à l'amélioration de l'ajustement du système arcflèche pour une performance optimale. Dans le même contexte, l'analyse de l'interaction arc-flèche a été poursuivie par la mesure de la vitesse de la flèche en sortie d'arc à l'aide d'un circuit électronique fixé sur la poignée. De plus, cette méthode originale a permis l'estimation de la quantité d'énergie de déformation au niveau de l'arc pendant le tir (Tuijn \& Kooi, 1992 ; Kooi \& Sparenberg, 1980).

Enfin, ces méthodologies (cinématique et dynamique) ont été complétées par l'étude des sollicitations musculaires de l'archer, à l'aide d'enregistrements électromyographiques de surface (sEMG) (Hennessy \& Parker, 1990; Clarys et coll.; Soylu et coll. 2006).

Un nombre réduit d'études ont été réalisées sur la conception et le comportement de l'arc (matériaux, utilisation ou non de stabilisateurs, déformation etc.). 
Peu d'entre elles permettent d'identifier l'influence de l'arc sur la précision du tir pour un même tireur (Amandeep et coll 2009; B.W. Kooi et J.A. Sparenberg, 1980)

L'objectif de la présente étude est de quantifier l'influence entre deux modèles d'arcs et de flèches, sur la précision du tir d'un unique athlète expert (3ème mondial, de ce fait avec un geste très reproductible dans des conditions identiques).

\section{Méthode}

L'expérimentation s'est déroulée sur la zone de tirà l'arc à l'intérieur de la Hall Maîgrot afin de s'abstenir des perturbations des flux d'air et de l'environnement extérieur. Un sujet expert, 3ème mondial, du Pôle INSEP Tir à l'Arc, choisi par l'entraîneur a participé volontairement à cette étude. Ce sujet avait pour consigne de réaliser 1 série de 12 tirs de flèches de type 1 (F1) puis 1 série de 12 flèches de type 2 (F2). Pour chaque série, les 12 flèches sont strictement identiques. Les tirs sont réalisés sur une cible placée à $70 \mathrm{~m}$ du tireur. Pour chaque tir, le sujet avait pour consigne de réaliser la meilleure performance possible (atteindre le centre de la cible, appelé zone jaune dans la suite du présent document). Chaque série de flèches a été tirée avec deux arcs (Arc1, Arc2) susceptibles être utilisées par le sujet en compétition.

La hauteur de la flèche sur l'arc avant le tir a été mesurée à l'aide d'un double mètre. Pour chaque tir, la hauteur de la flèche a aussi été mesurée à l'arrivée sur la cible. Un radar (Stalker ATS) était fixé sur un trépied $3 \mathrm{~m}$ derrière le sujet dans l'axe du tir. Ce radar mesurait la vitesse maximale de la flèche à la sortie de l'arc. L'étendue de mesure du radar est estimée entre 1.5 et 482.5 km.h-1 \pm 1.7 (StalkerC), 2006). L'angle entre la flèche et l'axe horizontal est estimé à partir des différents paramètres mesurés et des équations de la mécanique classique en conditions aérienne. Cette estimation néglige les effets aérodynamiques de la flèche en mouvement.

Pour chaque paramètre (vitesse maximale et angle de la flèche avec l'axe horizontal en sortie d'arc, hauteur de la flèche et à l'arrivée sur la cible), les test de Jarque Bera (1987) et Lilliefors (1967) permettent d'étudier la normalité des séries de données. Les tests Anova à deux voies et multi variés ont été utilisé afin d'identifier l'influence du type d'arc et de flèche sur chaque tir.

\section{Résultats}

L'étude de la distribution de chaque série de données correspondant aux 12 tirs, montre que les variables vitesse, angle, hauteur de tir suivent une loi normale à l'exception des tirs réalisés en condition Arc2F1. Ce phénomène s'explique 
par l'erreur de visée annoncée par le tireur le jour de l'expérimentation et le manque de tirs réalisés dans chaque série. La distribution de la série de données représentant l'angle de tir et la hauteur de tir sur la cible redevient normale lorsque le tir défectueux est exclu.

L'analyse statistique Anova à deux voies montre que la condition arc influence de manière significative la vitesse du tir $(\mathrm{p}=0)$, l'angle de tir $(\mathrm{p}=0)$ et la hauteur du tir sur la cible $(\mathrm{p}=0)$. La condition flèche influence de manière significative la vitesse du tir $(\mathrm{p}=0)$, l'angle de tir $(\mathrm{p}=0.003)$ et la hauteur du tir sur la cible $(p=0.003)$. L'interaction des deux conditions influences de manière significative la vitesse du tir $(p=0.0003)$ et l'angle de tir $(p=0.02)$. L'interaction des deux conditions n'influence pas de manière significative la hauteur du tir sur la cible $(\mathrm{p}>0.05)$.

Pour la suite de l'étude statistique, le tir défectueux est exclu des variables de la condition Arc2 F1, afin d'étudier l'influence des conditions flèches et arc.

Le test statistique Anova multi variée montre que la condition de l'arc et de la flèche influencent (fig. 1) de manière significative la vitesse du tir $(\mathrm{p}=0)$. Dans le cadre de l'utilisation de l'Arc 1, il n'existe pas de différence significative de la condition flèche sur la vitesse du tir (fig. 2). Dans le cadre de l'utilisation de l'Arc 2, la condition flèche influence de manière significative $(\mathrm{p}<0.05)$ la vitesse du tir (fig. 3).

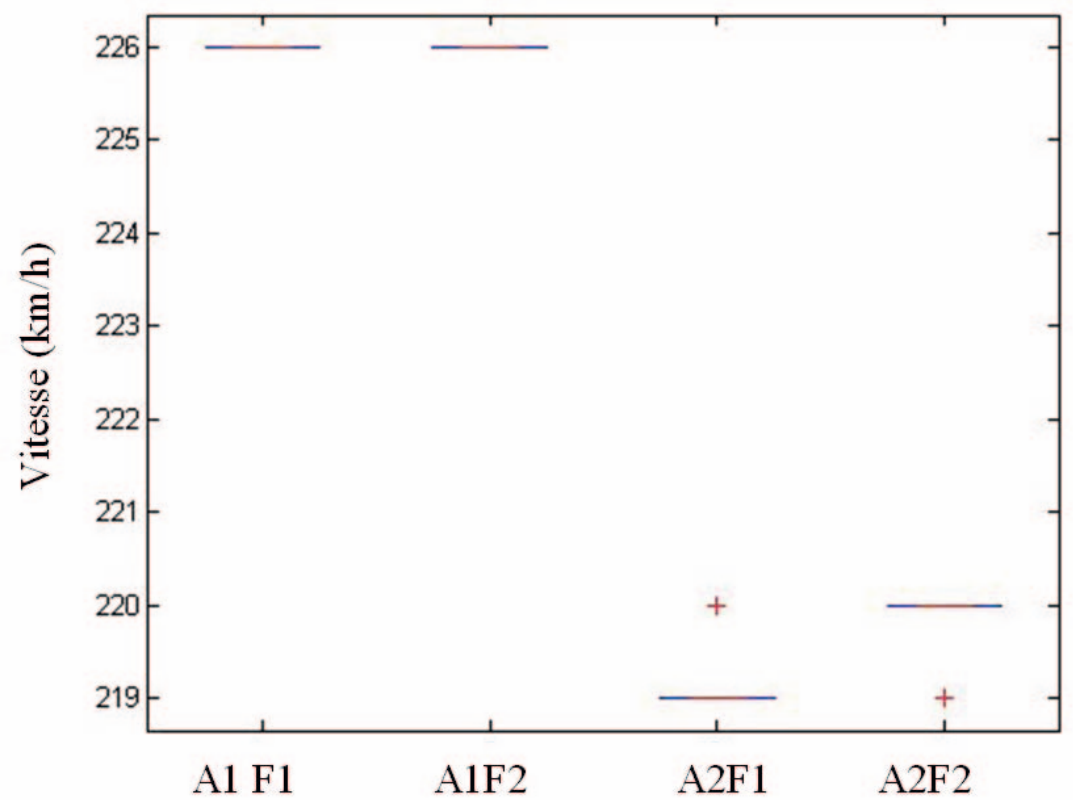

Figure 1. Moyenne et écart type de la vitesse du tir en fonction de la combinaison arc et flèche. 
Click on the group you want to test

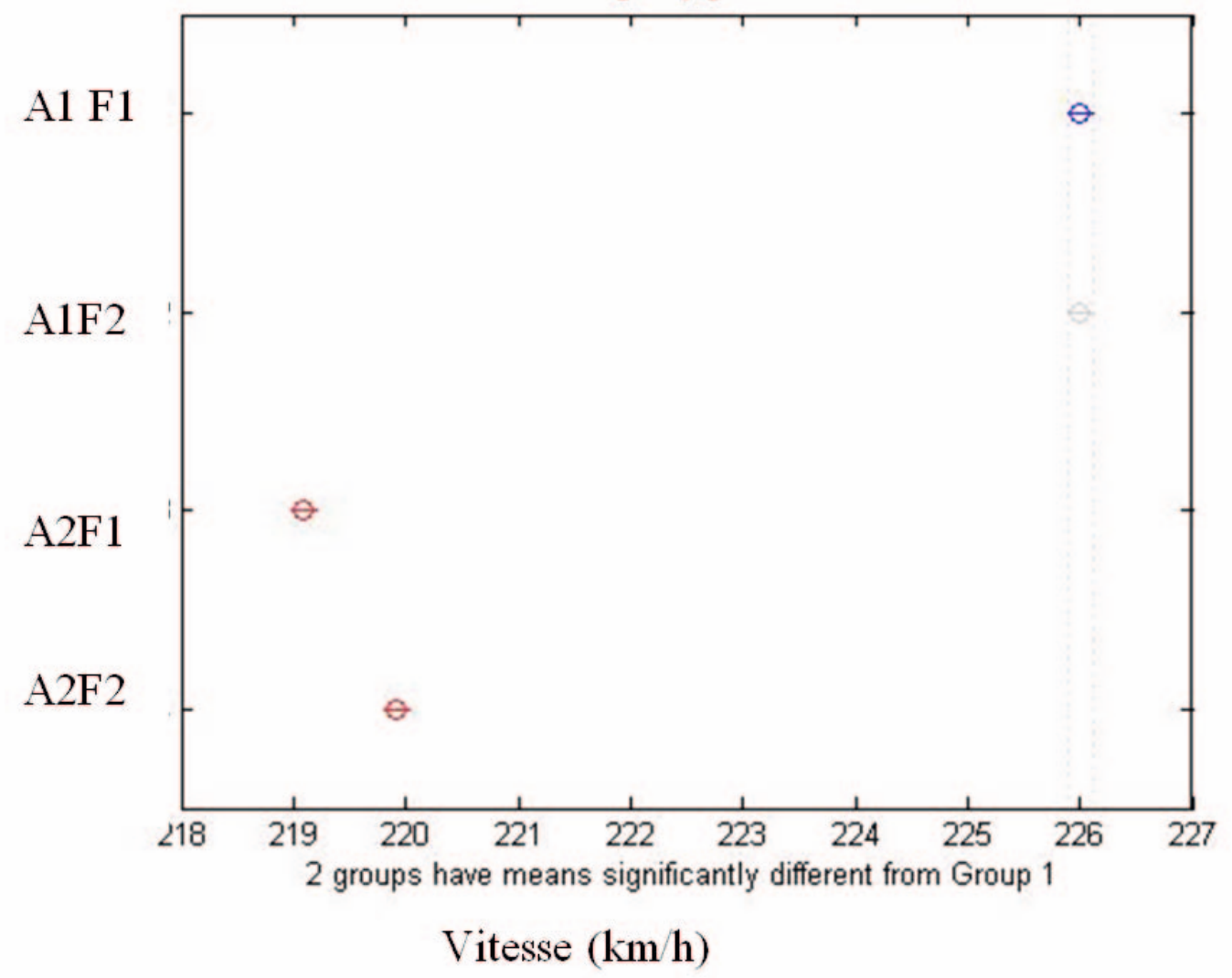

Figure 2. Différence non significative (en bleu) entre les conditions de tir 
Click on the group you want to test

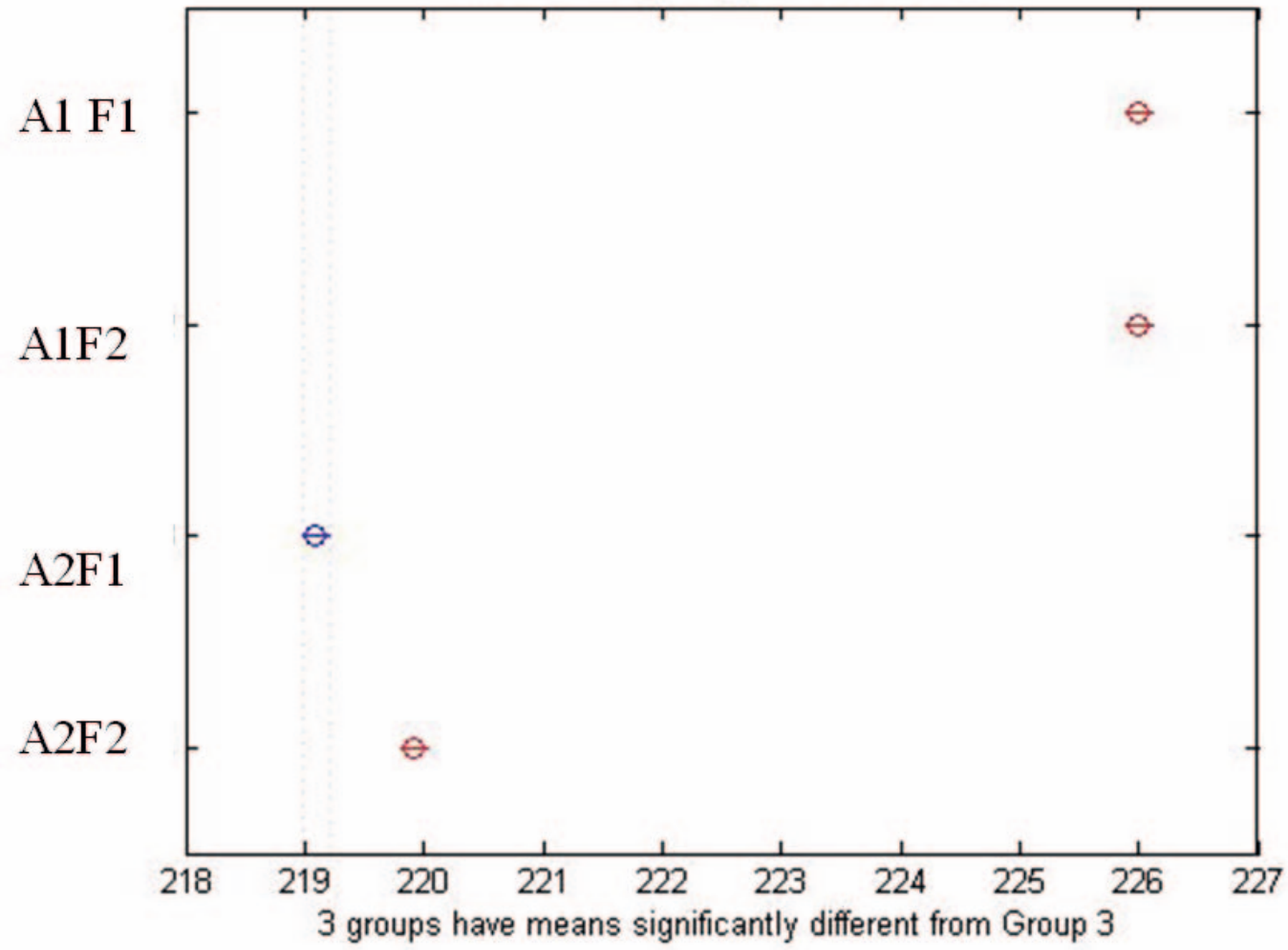

Figure 3. Différence non significative (en bleu) entre les conditions de tir

Le test statistique Anova multi variée montre que la condition de l'arc et de la flèche influencent (fig. 4) de manière significative l'angle de tir $(\mathrm{p}=0)$. Dans le cadre de l'utilisation de l'Arc 1, il n'existe pas de différence significative de la condition flèche sur l'angle de tir (fig. 5). Dans le cadre de l'utilisation de l'Arc 2, la condition flèche influence de manière significative $(\mathrm{p}<0.05)$ l'angle de tir (fig. 6). 
Sport Science Review, vol. XX, No. 1-2, April 2011

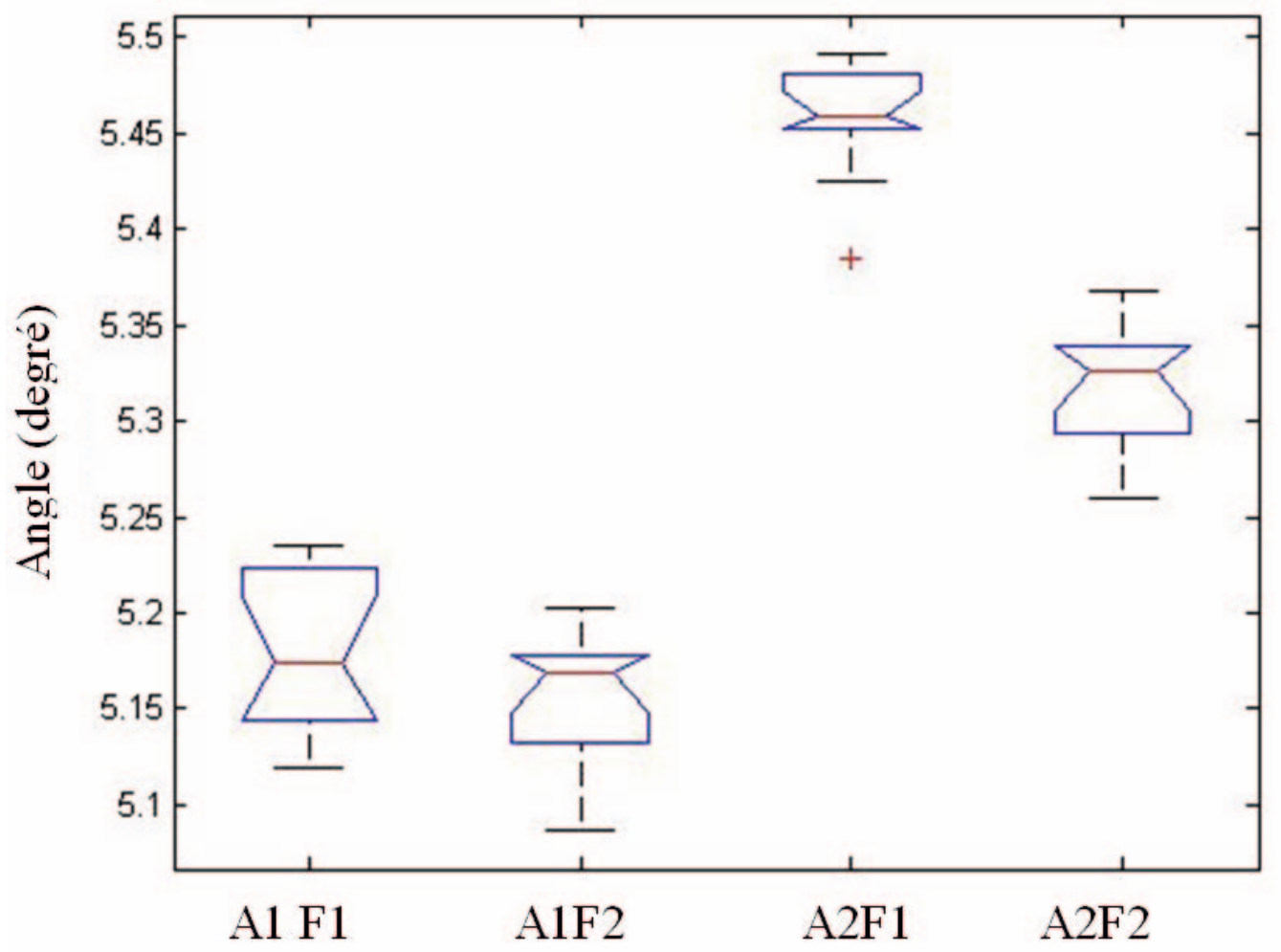

Figure 4. Moyenne et écart type de l'angle de vitesse du tir en fonction de la combinaison arc et flèche. 
Click on the group you want to test

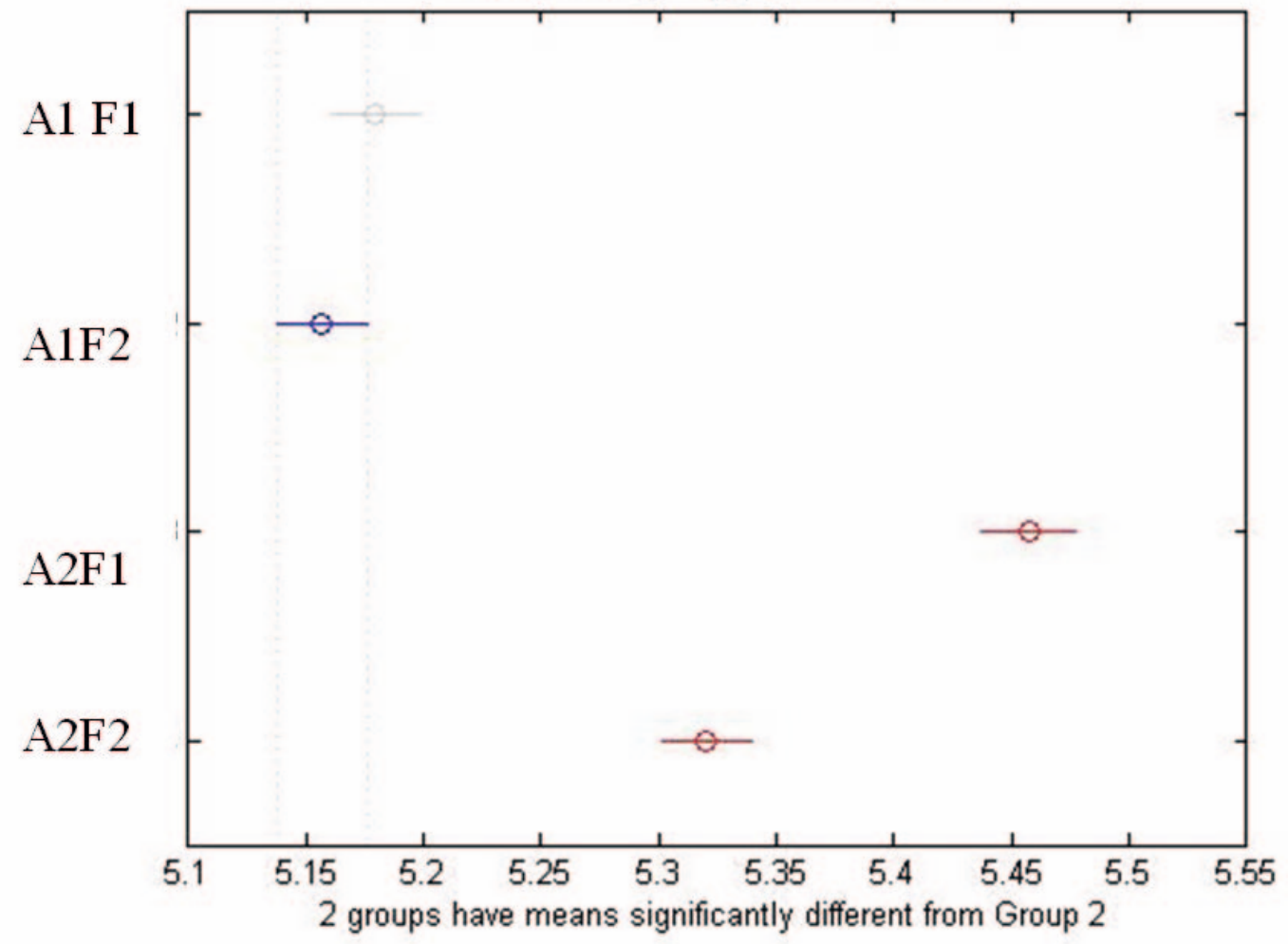

Angle (degré)

Figure 5. Différence non significative (en bleu) entre les conditions de tir 
Click on the group you want to test

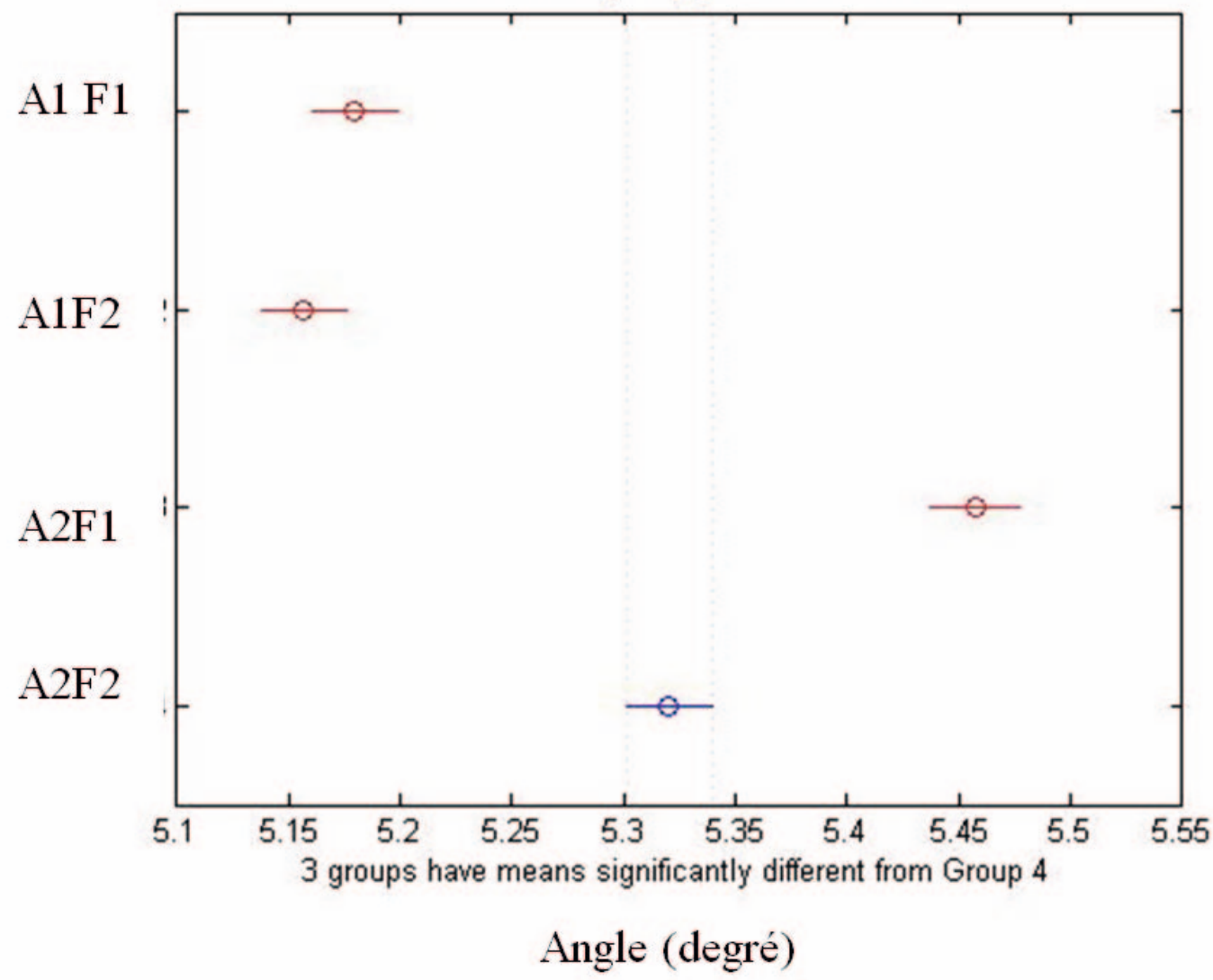

Figure 6. Différence non significative (en bleu) entre les conditions de tir

Le test statistique Anova multi variée montre que la condition de l'arc et de la flèche influencent (fig. 7) de manière significative la hauteur du tir sur la cible ( $\mathrm{p}=3.94 * 10-13)$. Dans le cadre de l'utilisation de l'Arc 1, il n'existe pas de différence significative de la condition flèche sur la hauteur du tir sur la cible (fig. 8). Dans le cadre de l'utilisation de l'Arc 2, la condition flèche influence de manière significative $(\mathrm{p}<0.05)$ la hauteur du tir sur la cible (fig. 9). La condition Arc2 flèche 1 n'est pas significativement différente des conditions utilisant l'arc1 en terme d'influence sur la hauteur du tir sur la cible. 
Modélisation du trajet global de la flèche

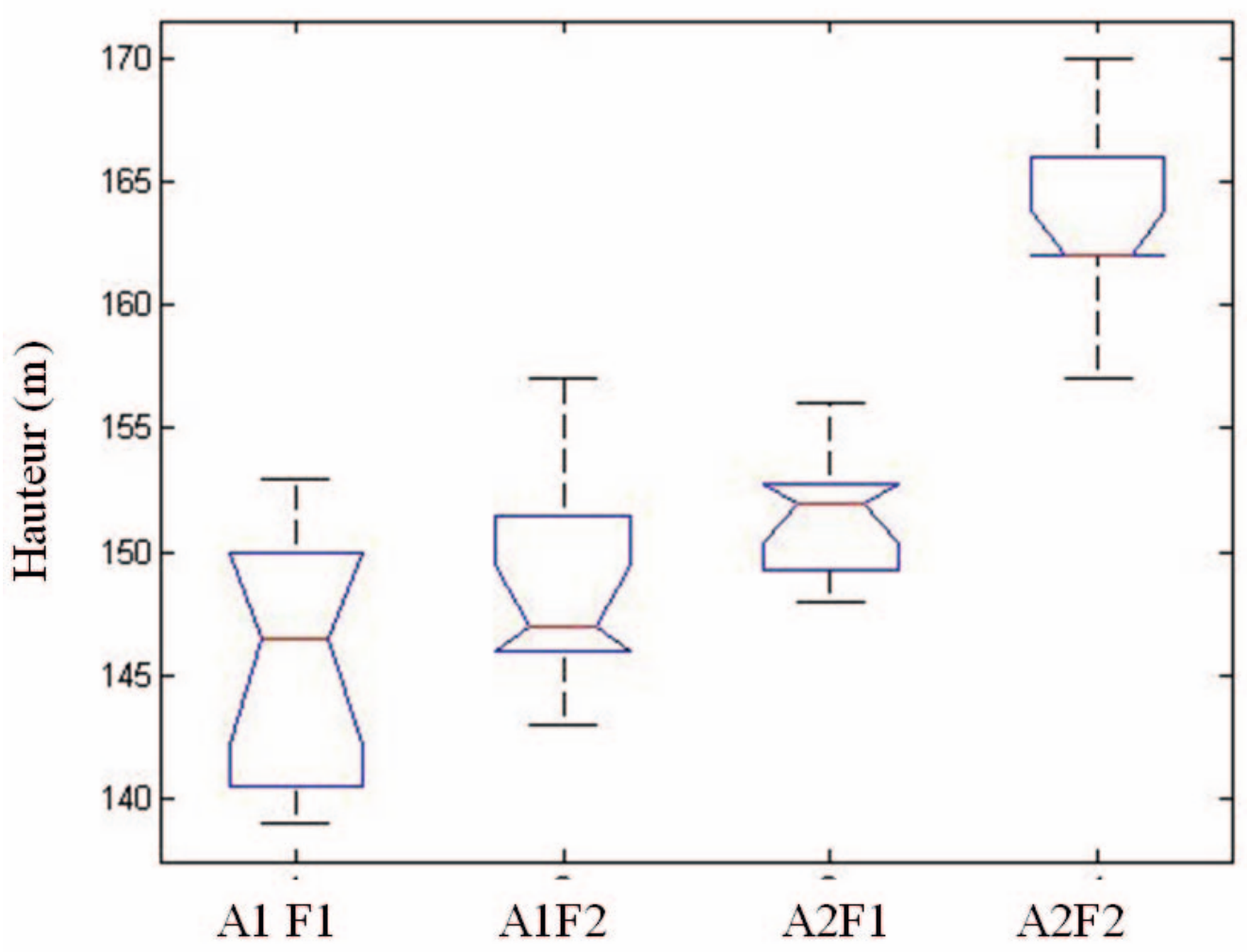

Figure 7. Moyenne et écart type de la hauteur du tir sur la cible en fonction de la combinaison arc et flèche. 
Sport Science Review, vol. XX, No. 1-2, April 2011

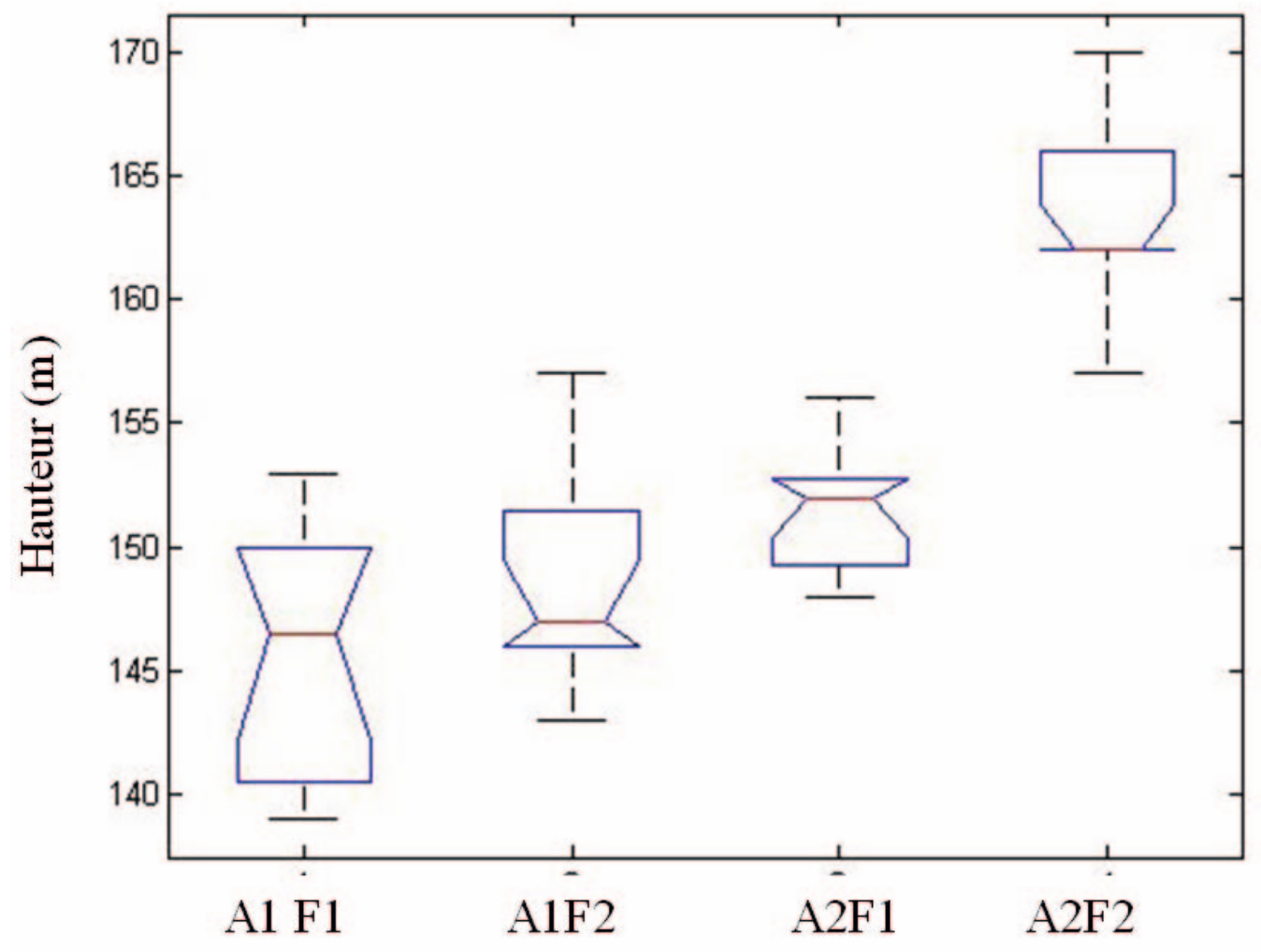

Figure 8. Différence non significative (en bleu) entre les conditions de tir 
Click on the group you want to test

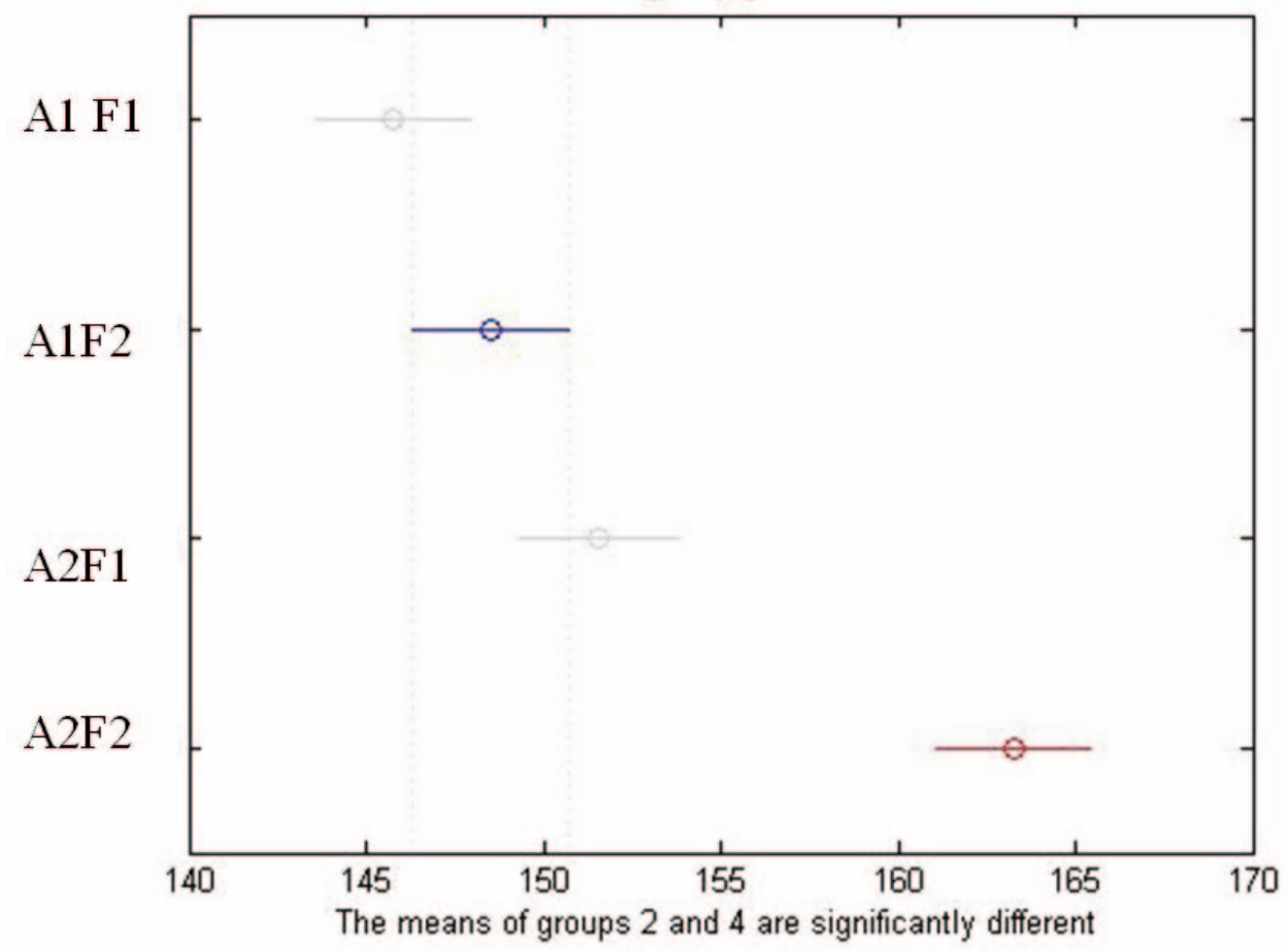

Hauteur du tir sur la cible (m)

Figure 9. Différence non significative (en bleu) entre les conditions de tir

\section{Discussion et conclusion}

L'interprétation de la figure 7 montre que la condition arc2 flèche2 (A2F2) semble la moins adaptée au tireur étudié dans le cadre de l'atteinte de la zone jaune de la cible dont la hauteur est comprise entre 131 et $154 \mathrm{~cm}$. L'interprétation des figures 8 et 9 montre que la condition arc2 flèche1 (A1F1) permettrait au tireur étudié d'atteindre la zone jaune de la cible avec autant de précision que les conditions utilisant l'arc1 (A1).

L'analyse des figures 1 à 6 montre que la condition arc2 flèche1 (A2F1) permettant d'atteindre la zone jaune de manière similaire aux conditions utilisant 
l'arc1 (A1), dan ce cas le tir est réalisé avec une vitesse plus faible $(219 \pm 0.3$ versus $226 \pm 0 \mathrm{Km} / \mathrm{h}$ ) et un angle plus élevé ( $5.45 \pm 0.3$ versus $5.16 \pm 0.03$ degré).

Les résultats présentés ici et leur interprétation restent soumis à la faible quantité de tir réalisé dans chaque condition ce qui réduit considérablement la validité statistique et l'hypothèse que la fatigue accumulée par le tireur pour la réalisation des tirs successifs n'affecte pas la précision au cours et entre les différentes séries.

Nous pouvons alors supposer que le tireur pourrait avoir intérêt à utiliser de manière préférentielle la condition arc2 flèche 1 (A2F1) avec un réglage spécifique pour l'arc 2. Dans cette condition, le tireur pourrait réaliser les tirs dans la zone jaune dite la plus performante avec un effort musculaire moindre pour bander l'arc2 (A2) que dans le cas de l'utilisation de l'arc 1 (A1). Dans les conditions expérimentales rencontrées, la combinaison arc 2 flèche 2 (A2F2) permettrait d'atteindre la cible de manière optimale (zone jaune) en conservant une plus grande plage d'amplitude d'angle de tir (0.6 degré) que dans le cas de l'utilisation de l'arc1 (0.08 degré).

La condition flèche n'influencerait pas la précision du tir de l'arc1 (A1). Par contre, la condition flèche influencerait la précision du tir de l'arc 2 (A2). La combinaison arc2 flèche 2 (A2F2) avec le réglage réalisé sur l'arc2 ne semble pas la plus adaptée pour le tireur étudié en vue d'atteindre la zone jaune. Il est possible qu'un changement de réglage de l'arc 2 en fonction de la flèche 2 pourrait permettre au tireur d'atteindre une précision similaire aux autres conditions.

\section{Références}

Amandeep, S., Virk, J., Summerscales, W., Hall, S. M., Grove, M. \& Miles, M. (2009). Design, manufacture, mechanical testing and numerical modelling of an asymmetric composite crossbow limb. Composites: Part B 40, 249-257

Avon-Coffrant, F. (1977). Le tir à l'arc: technique-entrainement-compétition. Paris: Amphora.

Clarys, J. P., Cabri, J., Bollens, E., Sleeckx, R., Taeymans, J., Vermeiren, M., Van Reeth, G., \& Voss, G., (1990). Muscular activity of different shooting distances, different release techniques, and different performance levels, with and without stabilizers, in target archery. J Sports Sciences, 8(3), 235-57. 
Ferriou, C., Flute, S., \& Frere, L. (1992). Tir à l'arc: découverte, technique, entraînement. Paris: Laffont

Frederic, L. (1979). Le tir à l'arc. Paris: Laffont

Hennessy, M. P., \& Parker, A. W. (1990). Electromyography of arrow release in archery. Electromyogry Clinical Neurophysiology, 30(1), 7-17.

Hunt, P., Libert, P., Mallet, H. (1987). Le tir à l'arc, sport olympique: matériel, technique, pédagogie. Paris: Bornemann

Kooi, B. W., (1998). Bow and arrow interaction in archery. Journal of Sports Sciences, $16,721 \pm 731$.

Kooi, B. W., \& Sparenberg., J. A. (1980). On the static deformation of a bow Journal of Engineering Mathematics, 14(1), 27-45

Leroyer, P., \& Le Gaverec, M. (1991). Le tir à l'arc. Paris: Bornemann.

Nayrole, M., \& Simon, F. (1990). Le tir à l'arc. Paris: Denöel.

Rousseau, J., \& Nicolier, E. (2005). Les fondamentaux du tir à l'arc: Initiation et perfectionnement. Paris: Amphora.

Soylu, A. R., Ertan, H., \& Korkusuz, F. (2006). Archery performance level and repeatability of event-related EMG. Human Movement Sciences, 25(6), 767-74.

Tuijn, C., \& Kooi, B. W. (1992). The measurement of arrow velocities in the students' laboratory. European Journal of Physics, 13, 127-134. 
Daniel DINU is employed as full-time researcher/engineer, Ph.D., at the National Institute for Sport, Expertise and Performance, Paris, France. His research areas are: Biomechanics; 3D modeling of the specific rigid bodies connected by joints and using an anthropometric model with an articulated trunk; Peripheral expression of the motor activity based on the analysis resulting from the video graphic, infra-red optoelectronic, inertial navigation technology and electromyography data. Since 1995, heworked with the best French throwers and gymnastics in the aim to improve the athlete's performance and motion in sport.

Nicolas HOUEL, is an associate scientific assistant, $\mathrm{PhD}$ in Biomechanics, at the $\mathrm{Na}$ tional Institute of Sport, Expertise and Performance (INSEP). His is specialized in biomechanics and accuracy of measurement in human motion. Since 2000, he worked with national teams of swimming and Nordic skiing in the aim to improve the athlete's performance and motion in sport.

Marc DELLEMBACH, is a national trainer for archer team. He works at the National Institute of Sport, Expertise and Performance (INSEP) has head of trainer of the national group of archers for the French archer's association. He trains some of the best national archers. 